\title{
Solar confocal interferometers for sub-picometer-resolution spectral filters
}

\author{
G. A. Gary ${ }^{1}$, C. Pietraszewski ${ }^{2}$, E. A. West ${ }^{1}$, and T. C. Dines ${ }^{2}$ \\ 1 National Space Science and Technology Center, Marshall Space Flight Center, Huntsville, AL, 35812, USA \\ e-mail: Allen.Gary@nasa.gov \\ 2 IC Optical Systems Ltd., Beckenham, Kent, BR34TW, UK \\ e-mail: sales@icopticalsytems.com
}

Received 22 August 2006 / Accepted 23 January 2007

\begin{abstract}
Aims. The confocal Fabry-Pérot interferometer allows sub-picometer spectral resolution of Fraunhofer line profiles. Such high spectral resolution is needed to keep pace with the higher spatial resolution of the new set of large-aperture solar telescopes. The line-of-sight spatial resolution derived for line profile inversions would then track the improvements of the transverse spatial scale provided by the larger apertures. In particular, profile inversion allows improved velocity and magnetic field gradients to be determined independent of multiple line analysis using different energy levels and ions. The confocal interferometer's unique properties allow a simultaneous increase in both étendue and spectral power. The higher throughput for the interferometer provides significant decrease in the aperture, which is important in spaceflight considerations.

Methods. We have constructed and tested two confocal interferometers. A slow-response thermal-controlled interferometer provides a stable system for laboratory investigation, while a piezoelectric interferometer provides a rapid response for solar observations. Results. In this paper we provide design parameters, show construction details, and report on the laboratory test for these interferometers. The field of view versus aperture for confocal interferometers is compared with other types of spectral imaging filters. We propose a multiple etalon system for observing with these units using existing planar interferometers as pre-filters. The radiometry for these tests established that high spectral resolution profiles can be obtained with imaging confocal interferometers. These subpicometer spectral data of the photosphere in both the visible and near-infrared can provide important height variation information. However, at the diffraction-limited spatial resolution of the telescope, the spectral data is photon starved due to the decreased spectral passband.
\end{abstract}

Key words. Sun: atmosphere - instrumentation: interferometers - Sun: magnetic fields - Sun: general - Sun: infrared

\section{Introduction}

Solar physicists are beginning, on a regular basis, to spatially resolve below 100 kilometer on the sun by employing optical 1-m class, and larger, aperture telescopes (Schlichenmaier 2006; Bettonvil 2004; Keil et al. 2004; Bernasconi et al. 1999). It is conceivable to resolve to $\sim 10 \mathrm{~km}$ in the next couple of decades with semi-monolithic or interferometric telescopes (Davis et al. 2005; Hammerschlag et al. 2004; Damé 1994). These operational, under-construction, or conceived telescopes include Flare-Genesis $(0.8 \mathrm{~m})$, Themis $(0.9 \mathrm{~m})$, Swedish Solar Telescope $(1.0 \mathrm{~m})$, SunRise $(1.0 \mathrm{~m})$, McMath-Pierce $(1.5 \mathrm{~m})$, Gregor $(1.5 \mathrm{~m})$, Dutch Open Telescope++ $(1.5 \mathrm{~m})$, New Big Bear Solar Telescope (1.6 m), Advance Technology Solar Telescope $(4.0 \mathrm{~m})$, Magnetic Transition Region Probe $(6.0 \mathrm{~m})$ and Giant Solar Optical Telescope $(11.0 \mathrm{~m})$. This effort of larger apertures for improved spatial resolution has been conducted in unison with superior designs and techniques (e.g., improvements in (i) image stabilization by adaptive optics for ground-based observatories, (ii) thermal designs to reduce locally induced seeing effects, (iii) polarization resolution by lowering instrumental effects, (iv) the size of the CCD arrays to increase the field of view, and (v) imaging processing to allow improved data analysis). Radiative transfer theory has improved with advanced magnetohydrodynamical numerical simulations at the sub-granular scales to resolve elemental flux tubes. However, visible-infrared, high-spectral-resolution narrow-band imaging filters at a spectral resolution of $\Delta \lambda / \lambda \leq 10^{-6}$ have not received much attention. This spectral resolution is currently provided by the nonimaging Fourier Transform Spectrometer (FTS) on the National Solar Observatory (NSO)/McMatch-Pierce Telescope. Using the FTS and studying elemental flux tubes, Stenflo et al. (1984) point out that the spectral measurements can be independent of the unresolved spatial elements, and hence high spectral resolution is a key component to understanding the structure of the sun. Mathew et al. (2003) have used the 3 pm full width half maximum $(F W H M)$ spectral resolution Tenerife Infrared Polarimeter (TIP) and inverted the FeI $1.56 \mu \mathrm{m}$ Stokes profiles using the SPINOR code to obtain an estimate of the magnetic fields gradient in an umbral region. In general, the line-of-sight spatial resolution derived from profile inversions should follow the improvements in the transverse (i.e., image plane) spatial resolution with larger apertures; however this will require sub-picometer spectral resolution. It is clear that the use of several lines with different magnetic, thermal, and Doppler sensitivities will help resolve the vertical information. However this process has its problems associated directly with the sensitivity variation which is used, i.e. different mass, different ions, different transitions, and different Landé factors. Through using a single transition and exploring the depth by its variation of opacity with position in the line (or response function) we avoid these sensitivity differences. 


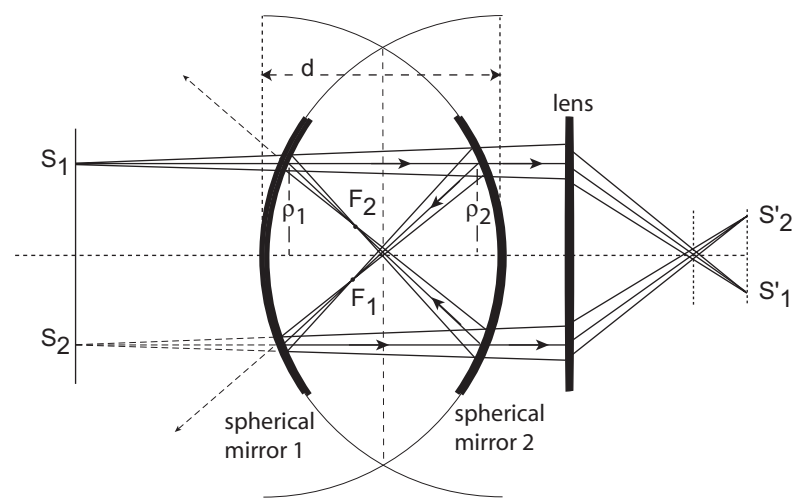

Fig. 1. The confocal optical configuration. In this configuration, the distance, $d$, between the two spherical mirrors (bold arcs) is set equal to the confocal distance, $r$, the radius of the spherical mirrors. To the upper left of the confocal mirrors, a real object is located at $S_{1}$. The transmitted light paths through the confocal mirrors and a lens are shown with the two resulting real images $S_{1}^{\prime}$ and $S_{2}^{\prime}$ formed by $2^{2 k}$ or $2^{2 k}+2$ mirror reflections, respectively. To show the symmetry of the optical path, the virtual object $S_{2}$ is shown. In this figure, the distance $\rho_{1}$ from the optical axis of the entrant ray at mirror 1 and its distance $\rho_{2}$ at mirror 2 are approximately equal. The two spherical mirror focal points are $F_{1}$ and $F_{2}$. The two reflected beams (oblique dash lines) result when the resonance interferometric condition is not satisfied. (After Johnson 1968.)

Furthermore we will show that the trade-off between the signalto-noise ratio and spectral resolution is acceptable. In this paper we present the spherical or confocal interferometer, an unnoticed technique in solar physics, which shows promise in combining high-spectral resolution and imaging, such that vertical gradients in mass advective flows and magnetic field gradients can be derived on a scale compatible with the horizontal gradients.

Normal or planar Fabry-Pérot interferometers (FPP) have a pair of mirrored parallel plates (etalons) and the light passes through the system when the gap between the plates is an integer number of the wavelength (Fabry and Pérot 1902; Vaughan 1989). The $F W H M$ spectral passband $\left(\Delta \lambda_{F W H M}\right)$ is dependent on the separation between the plates. These FPPs are used as standard narrow passband imaging filters in solar physics in the US Air Forces Optical Solar Patrol Network (OSPAN, $\Delta \lambda_{F W H M} \sim 10 \mathrm{pm}$ ), University of Hawaii's Mees Solar Magnetograph $\left(\Delta \lambda_{F W H M} \sim 7 \mathrm{pm}\right)$, Göttingen Astrophysical Institute's FPI $\left(\Delta \lambda_{F W H M} \sim 6.6 \mathrm{pm}\right)$, Arcetri Observatory's Interferometric BIdimensional Spectrometer (IBIS, $\Delta \lambda_{F W H M} \sim 2.3 \mathrm{pm}$ ), Kiepenheuer Solar Physics Institute's Triple Etalon Solar Spectrometer (TESOS, $\Delta \lambda_{F W H M} \sim 1.9 \mathrm{pm}$ ), and NSO/Sacromento Peak Observatory's dual etalon systems $\left(\triangle \lambda_{F W H M} \sim 1.7 \mathrm{pm}\right)$. For narrower $F W H M$ filters with larger gap distances, the confocal interferometer should be considered. A confocal (spherical) Fabry-Pérot interferometer (FPS) differs from the parallel-plate interferometer in that the mirrored etalons are identical spherical surfaces where the center of radius is at the surface of the other mirror. (Confocal means having the same foci.). Because of the spherical surfaces, the confocal interferometer can increase simultaneously both the spectral resolution and throughput and should be the preferred choice of interferometers for sub-picometer spectral filters (Hercher 1968; Vaughan 1989).

The path of the light through the confocal interferometer is typically a figure-eight shape (Fig. 1) with the exit beam re-entering the direction of the initial beam (to within paraxial approximation) (Connes 1958; Hercher 1968; Clark 1972;
Vaughan 1989). For the initial incident direction, the interference contributions to an exit ray come after $2^{2 k}$ reflections, where $k$ is a positive integer. The spherical mirrors and their associated thickness affect the final imaging and spectral resolution however we shall show this is not a significant effect. A main point of this paper is to describe the imaging quality of the confocal interferometers and the possible improvements that they could provide for solar physics, since the confocal interferometers have greater throughput and greater alignment tolerances than parallel plate interferometers under certain circumstances. An offset in confocal mirror alignment results in only a change in the optical axis of the system, when the confocal distance, $r$, is corrected by normal tuning. This results in a necessary alignment of the mirror centers to only the order of $\sim 0.1 D / r$; where as a planar Fabry-Pérot interferometer, the alignment tolerance for parallelism is on the order of $\sim \lambda / D$, where $\lambda$ is the wavelength and $D$ is the aperture.

One concern with increasing the spectral resolution is that this implies a deficit of photons as the spectral passband is reduced, causing longer exposure times. This is obviously problematic if the diffraction-limited spatial resolution is retained. For then the dependency of the size of the telescope is canceled since the light collected is a product of the telescope area and the spatial area sampled. If the aperture is varied it will be found that the product of the telescope's aperture area and the area of the diffraction-limited resolution element on the sun is constant. At the diffraction limit resolution, the exposure times are independent of the aperture size (Table 1, last column). Therefore, to collect a reasonable number photons for spectral analysis a reduced spatial resolution is require, as we will demonstrate. We will now consider the exposure times for two wavelengths, one in the visible and one in the near infrared.

The exposure time is determined by the time it takes to fill each detector pixel well with electrons. This time is governed by the well capacity of the CCD and the numbers of electrons generated each second by the light incident on each pixel. This latter parameter is itself determined by the quantum efficiency of the CCD and the number of photons incident each second on the pixel, $n_{\mathrm{p}}$. To determine $n_{\mathrm{p}}$, we consider the total radiant energy incident on a detector pixel per second, $P_{\mathrm{i}} . P_{\mathrm{i}}$ is equal to the solar radiant power per second per unit area per unit wavelength interval at the wavelength of interest, $I(\lambda)$, multiplied by the wavelength passband, $\Delta \lambda_{F W H M}$, the area of the telescope $A_{\mathrm{T}}$, and the effective area of a pixel on the sun, $f\left(A_{\mathrm{T}}\right)$, and corrected for transmission losses, $t_{\mathrm{r}}: P_{\mathrm{i}}=I(\lambda) \Delta \lambda_{F W H M} A_{\mathrm{T}} f\left(A_{\mathrm{T}}\right) t_{\mathrm{r}}$. The fraction of the total area collected by the telescope that is imaged on a detector pixel is equal to the ratio of solid angle of the projected pixel and the solid angle of the sun.

Observing at the telescope diffraction-limited resolution with the detector pixel size set by the Nyquist spatial frequency, the pixel size is half the diffraction-limited size. The projected solid angle of the pixel is then given by $\Omega_{\text {pixel }}=\pi\left(\frac{1.22 \lambda}{4 D_{\mathrm{t}}}\right)^{2}$. The solid angle of the sun is $\Omega_{\text {sun }}=6.8 \times 10^{-5} \mathrm{sr}$, hence $f\left(A_{\mathrm{T}}\right)=\frac{\Omega_{\text {pixel }}}{\Omega_{\text {sun }}}$. Assuming $\lambda=\lambda_{\mathrm{V}}=630.15 \mathrm{~nm}$, a telescope with an effective area of $A_{\mathrm{t}}=7854 \mathrm{~cm}^{2}$, (i.e., $D_{\mathrm{t}}=1 \mathrm{~m}$ ), gives $\Omega_{\text {pixel }}=$ $1.2 \times 10^{-13} \mathrm{sr}$, and $f\left(A_{\mathrm{T}}\right)=1.7 \times 10^{-9}$. In the continuum, the incident solar flux is $I\left(\lambda_{\mathrm{V}}\right)=1645 \mathrm{erg} \mathrm{cm}^{-2} \mathrm{~nm}^{-1} \mathrm{~s}^{-1}$ at the wavelength $\lambda_{\mathrm{V}}$. With optics providing a total optical transmittance of $t_{\mathrm{r}}=0.01$ and a filter of $\Delta \lambda_{F W H M}=1 \mathrm{pm}$, we have: $P_{\mathrm{i}}=$ $I(\lambda) \Delta \lambda_{F W H M} A_{\mathrm{T}} f\left(A_{\mathrm{T}}\right) t_{\mathrm{r}}=\left(1645 \mathrm{erg} \mathrm{cm}^{-2} \mathrm{~nm}^{-1} \mathrm{~s}^{-1}\right)(0.001 \mathrm{~nm})$ $\left(7845 \mathrm{~cm}^{2}\right)\left(1.7 \times 10^{-9}\right)(0.01)=2.2 \times 10^{-7} \mathrm{erg} \mathrm{s}-1$. At a wavelength of $\lambda_{\mathrm{V}}=630.15 \mathrm{~nm}$, each photon has an energy of $3.15 \times 10^{-12} \mathrm{erg}$, so there is a flux of $n_{\mathrm{p}}=6.9 \times 10^{4}$ photons per 
Table 1. The diffraction limit resolution for large solar telescopes using values in the text $(\lambda=630.2 \mathrm{~nm} / 1564.8 \mathrm{~nm})$.

\begin{tabular}{|c|c|c|c|c|}
\hline $\begin{array}{l}\text { Telescope aperture } \\
D_{\text {tel }} \\
(\mathrm{m})\end{array}$ & $\begin{array}{l}\text { Angular resolution } \\
\theta_{\text {diff }} \\
(\operatorname{arcsec})\end{array}$ & $\begin{array}{l}\text { Spatial resolution } \\
\Delta X \\
(\mathrm{~km})\end{array}$ & $\begin{array}{l}\text { Number of diffraction } \\
\text { elements per arcsec }\end{array}$ & $\begin{array}{l}\text { Full-Well-Depth Exposure Times at } \\
\text { Diffraction Limit with } 1 \mathrm{pm} F W H M \\
\text { (s) }\end{array}$ \\
\hline 1 & $0.16 / 0.39$ & $115 / 285$ & $39 / 6$ & $0.16 / 0.02$ \\
\hline 2 & $0.08 / 0.20$ & 57 / 142 & 159 / 26 & $0.16 / 0.02$ \\
\hline 4 & $0.04 / 0.10$ & $29 / 71$ & $639 / 103$ & $0.16 / 0.02$ \\
\hline 6 & $0.03 / 0.06$ & $19 / 48$ & $1431 / 232$ & $0.16 / 0.02$ \\
\hline
\end{tabular}

second on a pixel. With a 0.8 quantum-efficiency $(\mathrm{QE})$ detector the number of electrons per sec is determined $\left(n_{\mathrm{e}}=5.6 \times 10^{4}\right)$. For a signal to noise of $S / N=1000$, the number of electrons needed to be collected is a million. Hence, the exposure time is $18 \mathrm{~s}$. However for photometry we can use $2 \times 2$ pixel binning, i.e., a resolution element, and reduce the exposure time to $4.5 \mathrm{~s}$ to collect a million photons. In the near infrared at $\lambda_{\mathrm{IR}}=1564.85 \mathrm{~nm}$ where the diffraction limit spatial resolution is larger but the solar flux is lower, $I\left(\lambda_{\mathrm{V}}\right)=269 \mathrm{erg} \mathrm{cm}^{-2} \mathrm{~nm}^{-1} \mathrm{~s}^{-1}$; the photon flux is $n_{\mathrm{p}}=1.6 \times 10^{5}$ photons per second per pixel and the exposure time is $2.9 \mathrm{~s}$ with an IR 0.5 QE detector. At both wavelengths it is assumed that the pixel well depth is $W_{\mathrm{D}}=1 \times 10^{5} \mathrm{e}^{-}$. Assuming Poisson counting noise is the dominate noise, the detector digitation then must be able to resolve $\frac{1}{\left(W_{D}\right)^{1 / 2}}$, e.g., 10 bit A/D. Multiple reads of the CCD is necessary but we have assumed that the readout rate is a fraction of the exposure time, hence the readout is ignored. We also assumed that the dark current can be ignored when compared with the Poisson noise.

For one spectral position the exposure times are acceptable considering the solar dynamics time scales with respect to a diffraction-limited resolution element on the sun. However to obtain a high-spectral-resolution line profile at $1 \mathrm{pm}$ spectral resolution and at the diffraction limit, the exposure time become problematic. Stepping at an interval of $0.5 \mathrm{pm}$ over a spectral scan range of $30 \mathrm{pm}$, the photoelectrons for this line profile are obtained in $270 \mathrm{~s}$ at $\lambda_{V}$ and $171 \mathrm{~s}$ for $\lambda_{\mathrm{IR}}$. Of course, this is over the full field of view. To obtain the full Stokes profiles, the addition polarimetry requirements increase the total time by a factor of 6. Therefore, to obtain full Stokes profile scans in a time significantly shorter than the solar oscillation period, the spatial resolution must be larger than the diffraction limit. For extended, horizontal stratified regions (e.g., penumbral Evershed flow regions) where resolving the vertical stratification is important, the employment of larger than diffraction-limited resolution would be acceptable and allow shorter exposure times.

A main emphasis for imaging interferometry is to provide high spatial resolution compatible with the spectral line information to be derived. The larger the gap between the etalon plates in either the parallel or spherical interferometers, the higher the spectral resolution becomes, i.e. the smaller the spectral passband. Since the large ground-based and space-based solar telescopes, which are planned or being built, produce higher transverse spatial resolution on the scale of 10-200 km (Table 1); it is desirable and necessary to also increase the spectral power to isolate the solar physics to a comparable spatial scale in the vertical direction. That is to dissect the line profile structure of the line formation region (e.g., photospheric lines have a height of formation $F W H M \sim 200-500 \mathrm{~km}$ ). In particular to determine the vertical (or line-of-sight) gradients of flows and fields, to the same scale as the transverse gradients of the flows and fields, and to interrupt the corresponding line asymmetries. The pressure scale height for the photosphere is $\sim 150 \mathrm{~km}$ and the photon mean path is also on this scale.

Having high spectral resolution is important in determining the vertical gradients. Balasubramaniam \& Uitenbroek (2001) investigated the velocity gradients over the height of formation of the non-magnetic sensitive iron line FeI $557.6 \mathrm{~nm}$ (Landé factor of $g=0$ ). They showed with numerical modeling that a $0.5 \mathrm{pm}$ passband is required to resolve the $\sim 100 \mathrm{~m} / \mathrm{s}$ advective flows. A second example infers that a sub-picometer passband in the near infrared can determine the vertical magnetic gradient of the normal field. Sanchez Almeida (2006) showed theoretically that the vertical magnetic gradient can be derived from high spectral resolution observations of a single Zeeman sensitive line. As an example of this, the difference between the spectral Stokes $V$ profiles of a line formed within a magnetic field region with a decreasing gradient and of a line formed within a constant magnetic field is plotted in Fig. 2. Using the umbra $\mathbf{M}$ model of Maltby et al. (1986) with a magnetic field strength of $B=3000 \mathrm{G}$, velocities of $v_{\text {mic }}=1 \mathrm{~km} \mathrm{~s}^{-1}$ and $v_{\text {mac }}=0 \mathrm{~km} \mathrm{~s}^{-1}$, an inclination of $45 \mathrm{deg}$, and an azimuth of $45 \mathrm{deg}$, these magnetic field calculations were generated with vertical field gradients of $0,-3$ and $-6 \mathrm{G} / \mathrm{km}$. Independent of the polarization strength, these differences can be resolved with sub-picometer spectral resolution. Observationally, Mathew et al. (2003) used a profile inversion program to infer a vertical magnetic gradient from the TIP observations $\left(\Delta \lambda_{F W H M}=\sim 3 \mathrm{pm}\right)$ and obtained a value of $\sim-4 \mathrm{G} / \mathrm{km}$. With sub-picometer resolution, the magnetic gradient can be improved and compared with values from other Zeeman sensitive lines at higher heights.

Increasing the spatial resolution to well below $100 \mathrm{~km}$ has brought about a bogus concern that visible photospheric structure will be limited by photon diffusion to an optical mean-freepath, i.e. about one pressure scale height, due to smoothing by radiative transfer. If this were true, it would also effect the inversion of the line profile and hence set a limit on the useful spectral resolution. The argument was that the photon mean free path is $\sim 200 \mathrm{~km}$ for unity optical depth at $500 \mathrm{~nm}\left(\tau_{5}=1\right)$ (Allen 1973). Countering that argument, visible observations by Rouppe van der Voort et al. $(2004,2005)$ and Stein \& Nordlund's (2006) hydrodynamic simulations have shown that much smaller structures exist and a resolution of less than $100 \mathrm{~km}$ is required for the photosphere. Bruls \& von der Lühe (2001), in an analysis of radiative transfer effects on the visibility of small-scale structures, performed 2D non-LTE radiative transfer computations for thin flux sheets with widths ranging from 10 to $160 \mathrm{~km}$ in the solar photosphere. They demonstrated that such small structures could be observed as small scale variations of intensity and polarization. With the conclusion that the size limit where the photospheric structure cannot be observed (due to smoothing radiative transfer effects) must lie well below $10 \mathrm{~km}$. Synthesis of line profiles from dynamical flux tube simulations suggests that visible structure in Stokes $V$ will exist down to size scales of $5 \mathrm{~km}$ (Stein \& Nordlund 2006). Hence scales much smaller than 


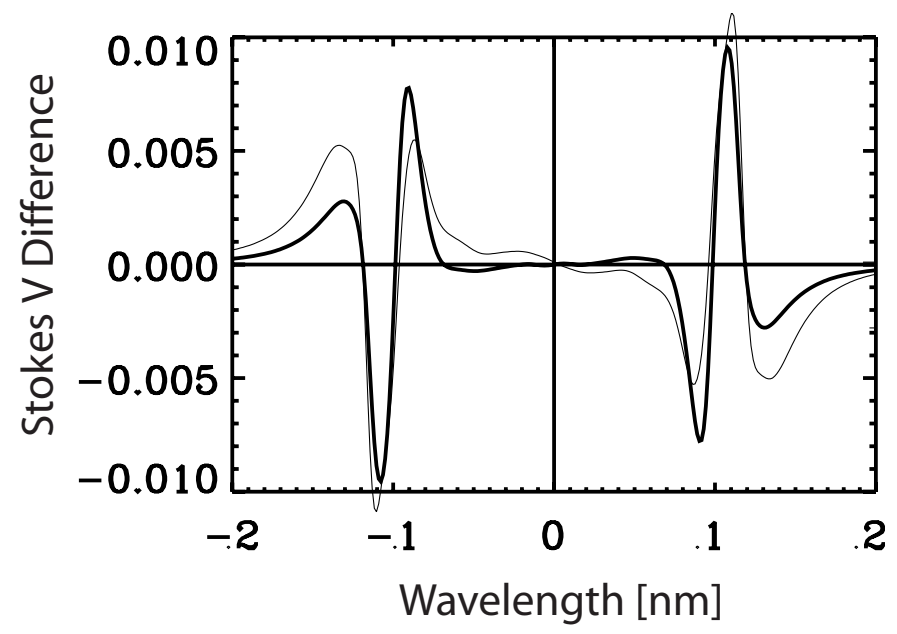

Fig. 2. For the infrared iron Zeeman line at $\lambda_{\mathrm{IR}}=1564.85 \mathrm{~nm}$, two differential Stokes V-profiles are shown. A differential profile is the difference between two Stokes profiles with one profile originating within the magnetic field with a vertical gradient and the other with a constant (zero gradient) magnetic field. The two different gradients plotted are $-6 \mathrm{G} \mathrm{km}^{-1}$ (thick line) and $-3 \mathrm{G} \mathrm{km}^{-1}$ (thin line). A sub-picometer resolution will discriminate between these models, with sufficient photons and polarimetry. For the visible line, $\lambda=\lambda_{\mathrm{V}}$, the splitting is a factor of $\left(\lambda_{V /} \lambda_{\mathrm{IR}}\right)^{2} \sim 1 / 6$ weaker, while the ratio of the splitting to the Doppler width is a factor of $\sim 1 / 3$, further requiring the sub-picometer spectral resolution. The $\lambda_{\mathrm{IR}}$ line width is $10 \mathrm{pm}$ (Solanki et al. 1992).

$100 \mathrm{~km}$ should be resolvable with imaging and spectral profile inversions.

In the next section we will compare the confocal interferometer with other spectral filters in terms of field of view and aperture size. Then we will provide initial design parameters, explain the construction details for two designs, and report on the laboratory test of these units. In the conclusion, we will propose a multiple etalon system for future testing the piezoelectric-controlled unit to obtain sub-picometer spectral profiles of photospheric in both the visible and near-infrared. These observations will employ existing planar Fabry-Perót interferometers as prefilters to the confocal system.

\section{Confocal interferometer comparison with other filters}

We will now compare the confocal interferometer with other imaging filter systems. For a 4-meter aperture telescope and for a given set of field of views (FOVs), Fig. 3 compares the optical aperture requirement for the confocal interferometer with an air-gap and solid planar Fabry-Pérot interferometer (FPI), a Lyot filter, and a Michelson interferometer. It shows that for a particular on-axis spectral resolution (e.g., $0.5 \mathrm{pm}$, dash horizontal line in upper panel) that the off-axis oblique chief ray at the incident angle produces an identical wavelength shift at the edge of the FOV of the same size as the on-axis resolution. For the particular filters this spectral shift is dependent on the refractive index of the gap or the particular type of interferometers. This typically sets the spectral resolution requirement for a collimated or telecentric optical system (Gary et al. 2003) and is due to the fact that the oblique chief ray angle in the interferometer sets the limits to the FOV for a given spectral resolution, via the Lagrange invariant or the ratio of the telescope aperture to the interferometer aperture. The confocal interferometer is similar to the Michelson interferometers in that it allows for a small
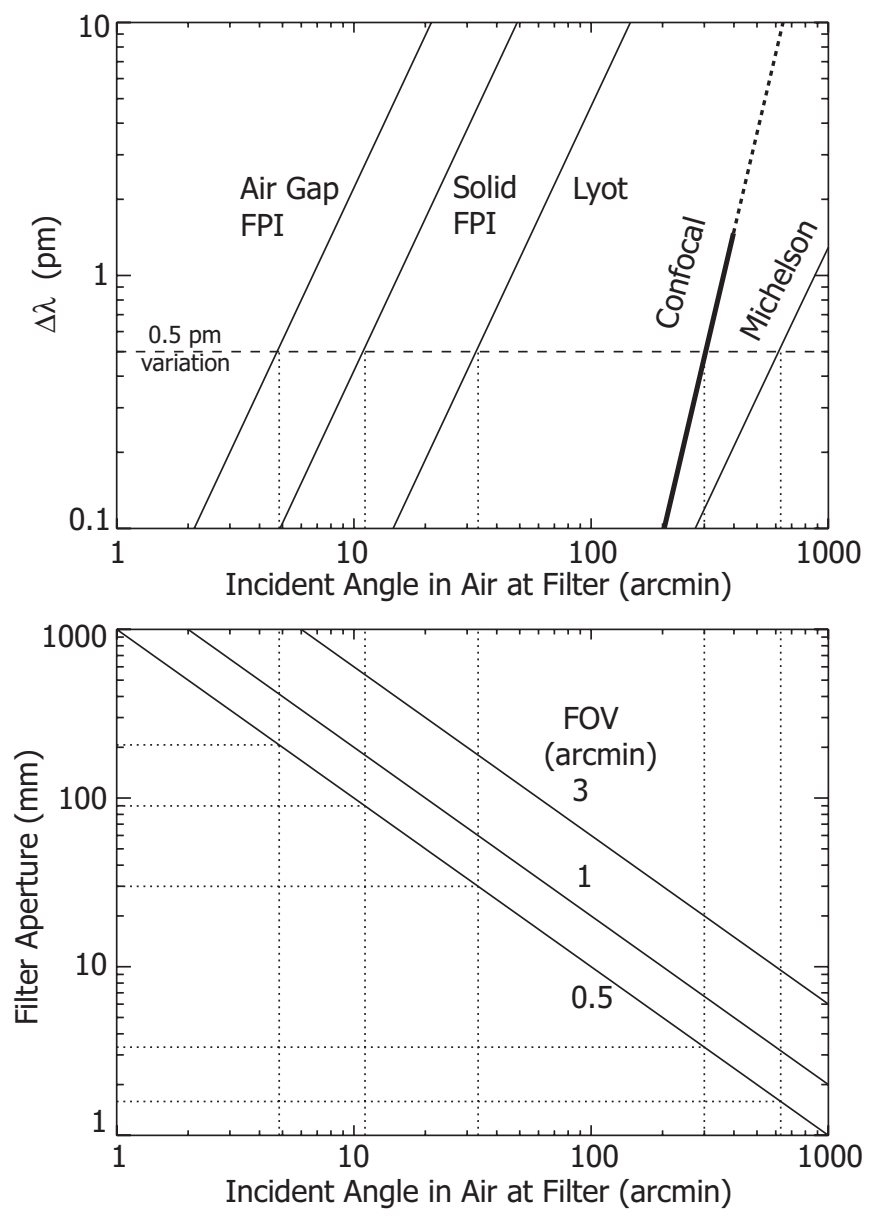

Fig. 3. The upper panel plots the wavelength shift $(\delta \lambda)$ as a function of the incident angle of the oblique chief ray for a collimated beam configuration for the set of filters discussed in the text. For the shift of $0.5 \mathrm{pm}$ the intercepts the various filter curves are plotted. Extrapolating these five-filter intercepts into the lower panel, the corresponding filter sizes for a 4-m telescope is given for three field of views (FOVs). In the upper panel, the confocal interferometer curve is shown as a dashed line where the aperture is smaller than $\sim 5 \mathrm{~mm}$, a manufacturable constraint. (After Gary et al. 2003.)

aperture interferometer, compared to the $100 \mathrm{~mm}$ plus etalons for the air gap etalon. The solid Michelson interferometer is designed for one wavelength while confocal interferometers can cover a wide range of wavelengths dependent on the reflectivity function of the etalon coatings. The relative wavelength shift, $\delta \lambda / \lambda$, as a function of the off-axis oblique chief-ray incident angle $\theta$ is given by $\delta \lambda / \lambda \sim\left|K \theta^{2} / 2\right|$, where constant $K$ is $1,1 / n_{\mathrm{o}}{ }^{2}$, $\left(n_{\mathrm{e}}-n_{\mathrm{o}}\right) / 2 n_{\mathrm{e}} n_{\mathrm{o}}{ }^{2},\left(p_{2} / n_{2}-p_{1} / n_{1}\right) /\left(p_{2} n_{2}-p_{1} n_{1}\right)$, and $\theta^{2} / 2$ respectively, for a air-gap Fabry-Pérot filter, a solid Fabry-Pérot filter, a wide field Lyot filter, a solid polarizing Michelson interferometer, and the confocal interferometer, where $\left(n_{\mathrm{o}}, n_{\mathrm{e}}, n_{1}\right.$, and $\left.n_{2}\right)$ and $\left(p_{1}\right.$ and $\left.p_{2}\right)$ are particular refractive indices and thicknesses (Gary et al. 2003; Hercher 1968). From these wavelength relationships, the confocal interferometer is nearly equivalent to the Michelson filters, and these have an advantage over an airgap Fabry-Pérot interferometer because a larger effective field of view can be obtained, or, equivalently, a smaller aperture can be used with a given field of view. The decrease in aperture for an air-gap interferometer is a factor of $\sim 2$ for a solid-gap $\left(\mathrm{LiNbO}_{3}\right.$ ) etalon, a factor of $\sim 10$ for a wide field, birefringent, Lyot filter with split-calcite elements, and a factor of $\sim 100$ for a confocal and a polarizing Michelson interferometer. However, 
for the ability to make multi-wavelength observations at subpicometer spectral resolution, the advantage goes to a confocal interferometer.

\subsection{On-axis modeling the confocal interferometer}

The characterization of a parallel plate Fabry-Pérot interferometer (FPP) for a telecentric beam configuration gives an effective $F W H M$ dependent on the maximum angle of incident $(\theta)$, while for a collimated beam configuration there is a variation of line center across the FOV. The single etalon equations for the central wavelength $\lambda_{\mathrm{o}}$, free spectral range $F S R$, total finesse $F$, reflectance finesse $F_{\mathrm{R}}$, integer order number $m$, and passband $\left(\Delta \lambda_{F W H M}\right)$ are given by the well-known relations: (i) $\lambda_{\mathrm{o}}=2 n \bar{d}$ $\cos (\theta) / m$, (ii) $F S R=\lambda_{\mathrm{o}}^{2} / 2 n \bar{d} \cos (\theta)$, (iii) $F_{\mathrm{R}}=\pi R^{1 / 2} /(1-R) \approx$ $-\pi / \ln R$, and (iv) $\Delta \lambda_{F W H M}=F S R / F$, where $\bar{d}$ is the etalon gap distance for parallel plates, $n$ is the refractive index of the gap, $R$ is the coating reflectance, and assuming no optical defects, i.e., $F=F_{\mathrm{R}}$ (Vaughan 1989). For small angles the wavelength shift is $\delta \lambda=\lambda_{\mathrm{o}} \theta^{2} / 2$ (Fig. 3). These inter-relationships determine the specific requirements for the parallel-plate filter. In particular for the planar Fabry-Pérot the ratio of the spectral resolution $\left(N_{\lambda}=\lambda / \Delta \lambda_{F W H M}\right)$ to the étendue $\left(U_{\mathrm{FPP}}=\pi D^{2} \lambda / 4 \bar{d} F_{\mathrm{R}}\right)$ is given by $N_{\lambda} / U_{\mathrm{FPP}}=4 \pi\left(\bar{d}^{2} / 2 D^{2}\right)\left(2 F_{\mathrm{R}} / \pi \lambda\right)^{2}$, where $D$ is the aperture size (Herscher 1968; Vaughan 1989). Increasing the gap distance increases the spectral power $N_{\lambda} \sim \bar{d}$ but decreases the étendue $U_{\mathrm{FPP}} \sim 1 / \bar{d}$.

Setting the distance between mirror centers, $d$, to the exact confocal separation, $r$, the corresponding on-axis, paraxial formulae for the confocal (spherical) Fabry-Pérot interferometer $(F P S)$ are: (i) $\lambda_{\mathrm{o}}=4 \mathrm{nr} / \mathrm{m}$, (ii) $F S R=\lambda_{\mathrm{o}}^{2} / 4 n r$, (iii) $F_{\mathrm{R}}=\pi R /\left(1-R^{2}\right)$, and (iv) $\Delta \lambda_{F W H M}=F S R / F$ (Vaughan 1989). The useable aperture or pupil spot radius is given by $\rho_{\mathrm{S}}=\left(r^{3} \lambda / F_{\mathrm{R}}\right)^{1 / 4}$, assuming the $F W H M$ at this edge is within $2^{-1 / 2}$ of the central FWHM (Vaughan 1989). If $\Omega$ is the beam solid angle, $\mathrm{A}$ is the usable aperture area, and the refractive index is set to unity, $n=1$, the confocal étendue is given by $U_{\mathrm{FPS}}=A \Omega=\left[\pi \rho_{\mathrm{s}}^{2}\right]\left[\pi \rho_{\mathrm{s}}^{2} / r^{2}\right]=2 \pi^{2} r \lambda / F_{\mathrm{R}}$ and the spectral power $N_{\lambda}=\lambda / \Delta \lambda_{F W H M}=4 r F_{\mathrm{R}} / \lambda$; hence the ratio of the resolving power to étendue for the confocal Fabry-Pérot interferometer is given by $N_{\lambda} / U_{\mathrm{FPS}} \sim 0.7\left(2 F_{\mathrm{R}} / \pi \lambda\right)^{2}$. Hence both the resolving power $\lambda / \Delta \lambda_{F W H M} \sim r$ and the étendue $U_{\mathrm{FPS}} \sim r$ increases with the confocal separation distance. For a fixed finesse, the confocal interferometer can increase simultaneously, both, the spectral power and throughput. For the same FSR and for $r \sim \bar{d} / 2$, the FPS becomes a better choice than the FPP when $r \sim 40 \mathrm{~mm}$ because of the defect finesse (Hercher 1968). For the FPP parameters, $\bar{d}>2^{1 / 2} D$, the étendue relation $U_{\mathrm{FPS}}>U_{\mathrm{FPP}}$ states that the confocal interferometer has greater light collecting property (Vaughan 1989, p. 194). For large-aperture solar telescopes, the critical result is that a smaller aperture can be used for the same number of photons passing through.

We have designed and built two sub-picometer confocal interferometer both performing in the visible and near infrared. Each FPS was designed with a $\Delta \lambda_{F W H M}=0.13 \mathrm{pm}$ $(F S R=2 \mathrm{pm})$ at $\lambda_{V}=630.2 \mathrm{~nm}$ and $\Delta \lambda_{F W H M}=0.8 \mathrm{pm}$ $(F S R=12 \mathrm{pm})$ at $\lambda_{\mathrm{IR}}=1564.85 \mathrm{~nm}$. This allows the solar FeI Zeeman sensitive lines at $\lambda_{V}$ and $\lambda_{\mathrm{IR}}$ to be scanned and allows a frequency stabilized HeNe laser line $\left(\lambda_{\mathrm{HeNe}}=632.8 \mathrm{~nm}\right)$ to be employed for laboratory test. The relationship between FSR, $F W H M$, reflectance, and confocal separation is shown in Fig. 4 for the visible and near infrared lines. Although the plot of reflectance versus FSR and FWHM is independent of the wavelength, we have separated the line plots into two reflectance

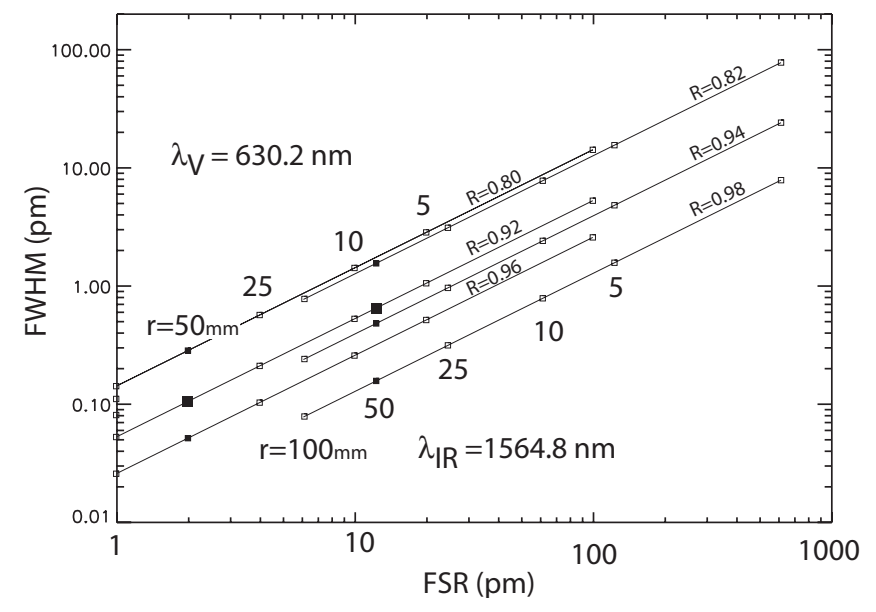

Fig. 4. The relation between the $F W H M$ and the FSR as a function of confocal separation $(r)$ and reflectance $(R)$ is plotted. The confocal separation distances $r$ in millimeters associated with $\lambda_{V}$ or $\lambda_{\mathrm{IR}}$ are seen at the top or bottom, respectively, of the set of constant reflectance curves. For the particular reflectances, the separations are shown as small boxes. For clarity, the confocal separation distances of the two wavelengths are, in general, on separate reflectance curves. The solid filled squares are for $r=50 \mathrm{~mm}$, and the two largest fill squares (for $\lambda_{V}$ at the left and $\lambda_{\mathrm{IR}}$ at the right) are associated with the confocal design of $R=0.92$ and $r=50 \mathrm{~mm}$.

groups in order to show their correlation with the confocal separation distances for $\lambda_{V}$ and $\lambda_{\mathrm{IR}}$. For the two wavelengths, the largest two squares plot the corresponding design values for $R=$ 0.92 for the same separation distance, $r=50 \mathrm{~mm}$. The set of box symbols at the ordinate $F S R=1 \mathrm{pm}$ refer to a separation of $r=$ $100 \mathrm{~mm}$ for $\lambda_{V}$. The reflectance finesse is $F_{\mathrm{R}}=\pi R /\left(1-R^{2}\right)=18.8$ and the resolving power is $N_{\lambda}=\Delta \lambda_{F W H M} / \lambda=4 F_{\mathrm{R}} r / \lambda=5 \times 10^{6}$ for $\lambda_{V}$ and $2 \times 10^{6}$ for $\lambda_{\mathrm{IR}}$. This implies a confocal separation distance of $r=50 \mathrm{~mm}$ for sub-picometer spectral resolution, and for ease of manufacturing the first units for testing have an aperture of $25 \mathrm{~mm}$. Because of the root-mean-square (rms) irregularities and roughness of the spherical surfaces, the total finesse is a combination of the reflectance finesse $F_{\mathrm{R}}$ and the defect finesse $F_{\text {defect }}$. For a rms irregularity of $\delta_{\text {rms }}=\lambda / \eta$, the defect finesse is $F_{\text {defect }}=\eta / 2$ (Hercher 1968). Hence to have $F_{\text {detect }}$ to match the $F_{\mathrm{R}}(R=0.92)$ then $\eta=38$ and $\delta_{\text {rms }}=\lambda / 38$, which is a reasonable smoothness for the spherical etalons. For these values the total finesse $F$ is then $F=\left(1 /\left(1 / F_{\mathrm{R}}^{2}+1 / F_{\text {defect }}{ }^{2}\right)\right)^{1 / 2}=2^{-1 / 2} F_{\mathrm{R}}=13.3$. This is an effective reflectivity of 0.88 . The $F W H M$ design values become $\Delta \lambda_{F W H M}=0.15 \mathrm{pm}$ and $0.90 \mathrm{pm}$. Inversely, if we had selected the total finesse to be 18.8, then the reflectance and detect finesse must be 26.5 which give $R=0.943$. Hence the final design reflectance value was set to be $R=0.95 \pm 0.02$ with surface figure to be better than $\lambda / 40$.

\subsection{Off-axis modeling the confocal interferometer and imaging theory}

An optical configuration for the confocal interferometer is shown in Fig. 1 within the paraxial approximation. The rays starting from $S_{1}$ after making quadruple (or $2^{2 k}$ ) reflections are reentrant with the original direction. After making two reflections or $2^{2 k+1}$, another set of rays emerge and form an additional image $\left(S_{2}^{\prime}\right)$. For each of the two beams, the FPS intensity is $50 \%$ that of a FPP, however, in principle, the second beam could be recombined or used. This second image appears to be coming from the virtual source at $S_{2}$. There are two beams that emerge back 
toward the source side (dashed arrows in Fig. 1). Clark (1972) gives the imaging relationships for an imaging confocal optical interferometer with the etalon plates with a radius of curvature given by $r$ and a separation of $d=r+e$, where $e$ is a small displacement. For $e=0$, the separation is the exact confocal distance. The phase difference for the rays is given by

$\delta_{1}=(2 \pi / \lambda)\left(\rho_{1}^{2} \rho_{2}{ }^{2} / r^{3}+2 e\left(\rho_{1}{ }^{2}+\rho_{2}{ }^{2}\right) / r^{2}\right)+\delta_{\text {on-axis }}$,

where $\rho_{1}$ and $\rho_{2}$ are the distances of the ray from the optical axis at the first and second spherical etalon surface, $\delta_{\text {on-axis }}=8 \pi(r+$ $e) / \lambda$ is the on-axis phase difference; and, for $\rho=\rho_{1}=\rho_{2}$, the off-axis phase difference is given by:

$\delta_{1}-\delta_{\text {on-axis }}=(2 \pi / \lambda)\left(\alpha^{4} r / 16+e \alpha^{2}\right)$,

where $\alpha=2 \rho / r$ (Vaughan 1989, Eqs. (5.6) and (5.7)). Using Fermat's principal, Clark (1972) determines the best focus, for a specific $\rho$, using the central diffraction angle $\alpha_{\mathrm{c}}$ for which $\partial\left(\delta_{1}\right) /$ $\partial \alpha=0$. This also sets the largest range of $\alpha$ for which the spectral information is passed and the associated spatial resolution. For input intensity $I_{0}$, the output diffraction intensity for the $S_{1}^{\prime}$ is given by

$I(\alpha)=\left(I_{0} / 2\right) /\left[1+(2 F / \pi)^{2} \sin ^{2}\left(\delta_{1} / 2\right)\right]$,

and $\alpha_{\mathrm{c}}=(-8 e / r)^{1 / 2}$. A plus and minus sign denotes if the wavelength is longer or shorter than the central peak. On the two sides of the intensity peak at HWHM (half width at half maximum), the phase difference is $\delta_{ \pm}=(2 \pi / \lambda)\left(-4 e^{2} / r \pm \lambda / F\right)+\delta_{\text {on-axis }}$, hence the cutoff angles become,

$\alpha_{ \pm}= \pm(-1)\left[-(8 e / r) \pm(16 \lambda / r F)^{1 / 2}\right]^{1 / 2}$,

which gives the variation of acceptable angles for which the passband is within FWHM. Hence if $\alpha_{-}=0$ then $\alpha_{+}=(64 \lambda / r F)^{1 / 4} \sim 2.8\left(\Delta \lambda_{F W H M} / \lambda\right)^{1 / 4}=5.15 \mathrm{deg}$. This is consistent with the angle $2 \rho_{\mathrm{S}} / r \sim 2\left(\lambda / r F_{\mathrm{R}}\right)^{1 / 4} \sim 3.6 \mathrm{deg}$, derived form Hercher's (1968, Eq. (17)) spot radius. Therefore the FOV is approximately $4 \mathrm{deg}$.

\section{Construction of a confocal interferometer}

For testing the image quality of the confocal interferometers, we have constructed two confocal interferometer using IC Optical Systems spherical mirrors of $r=r_{1}=50.065 \mathrm{~mm}$ internal confocal radius and external radius of $r_{2}=60.30 \mathrm{~mm}$. These $25 \mathrm{~mm}$ diameter fused silica plates $(n=1.459)$ have a $15 \mathrm{~mm}$ clear aperture and a plate thickness of $10.235 \mathrm{~mm}$. The measured mirror coating transmittances is $5.13 \%$ at $630.98 \mathrm{~nm}$ and $4.97 \%$ at $1564.2 \mathrm{~nm}$. The exterior surfaces, with anti-reflecting coatings for both wavelengths $(R \leq 0.25 \%)$, were made concentric with a radius of curvature of $60.25 \mathrm{~mm}$. By employing concave-convex etalon plates and the plate thickness, the optical power of each etalon plate is minimized for imaging quality and spectral resolution. The thick lens formula $f^{-1}=(n-1)\left(1 / r_{1}-1 / r_{2}+(n-\right.$ 1) $/ n r_{1} r_{2}$ ) gives a relative low-power, large focal length of $f \sim$ $-623 \mathrm{~mm}$ (while an optical Zemax code gives $f \sim-925 \mathrm{~mm}$ ).

In order to calibrate the etalon we need to be able to scan $j$-free spectral ranges, i.e., the confocal displacement should be $\delta d_{j}=j \lambda / 4 n$, a few microns. The spectral resolution should be $\sim 1 / 10$ th of the $\Delta \lambda_{F W H M}$, or about $(\lambda / 4 n) /(10 F)$. To infinitesimally displace the mirrors by this amount, the two confocal interferometers were designed to have good mechanical and environmental stability (Fig. 5). The piezoelectric-controlled interferometer follows the design by Budker et al. (1999) (cf., Vaughan 1989, Sect. 5.5.1). The thermally-controlled FPS design was engineered to allow initial precise displacement near the confocal separation.

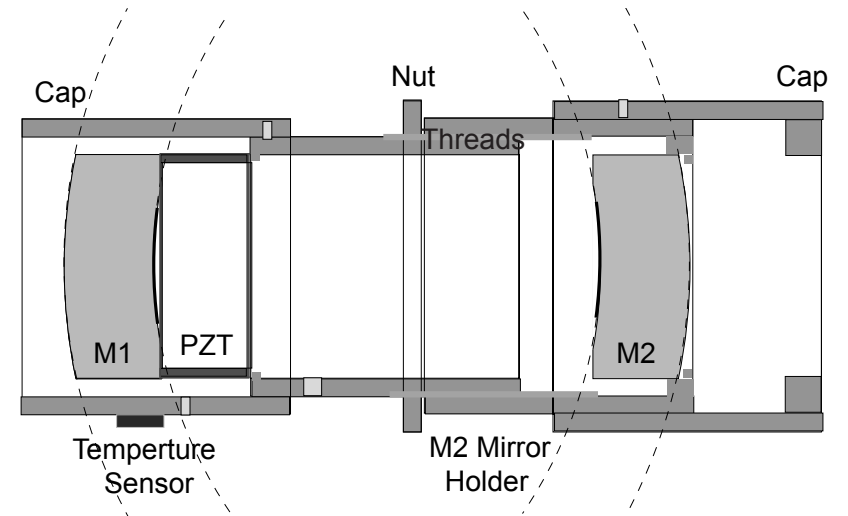

Piezoelectric-Controlled Confocal Interferometer



Thermally-Controlled Confocal Interferometer

Fig. 5. Schematic of both of the confocal Fabry-Pérot interferometers. Two sets of lines (dotted) shown have radii of confocal radii $R_{1}=50.065$ and $R_{2}=60.30$ with their radii centered at the center of interior mirror surfaces M1 and M2.

\subsection{Piezoelectic-Tunable FPS}

The piezoelectric controller utilizes a thin-wall hallow cylinder of lead zirconate titanate $\left(\mathrm{PbZr}_{0.52} \mathrm{Ti}_{0.48} \mathrm{O}_{3}, \mathrm{PZT}\right)$ made of Channel Industries C-5700 and milled by Boston Piezo Optics to a length of $10 \mathrm{~mm}$, outside diameter $25 \mathrm{~mm}$, and wall thickness of $1 \mathrm{~mm}$, and nickel plated (Fig. 7, lower panel). For a voltage $V$, the linear displacement along the cylindrical axis is given by $\Delta d=d_{31} L V / t$, where $d_{31}=-0.25 \mathrm{~nm} /$ volt is the piezoelectric constant, $L$ is the length of the cylinder, and $t$ is the wall thickness of the cylinder; then $\Delta d=2.5 \mathrm{~nm} \cdot V$ or $\Delta d=4.1 \times 10^{-3} \lambda V$ at $\lambda_{V}=630.2 \mathrm{~nm}$. A voltage of 60 volts allows tuning through one free spectral range for the visible line. For a finesse of $F=13$, then the displacement resolution of the tube should be $1.2 \mathrm{~nm}$ or $\sim \lambda / 500$ with a voltage resolution of $\sim \frac{1}{2}$ volt. The coefficient of thermal expansion led to a displacement of $\sim 40 \mathrm{~nm} / \mathrm{C}$, hence the temperature needs to be stable or known to within $\sim 0.1 \mathrm{C}$. The calibration between voltage and displacement is required to correct for hysteresis and nonlinearity (Fig. 8).

The construction schematic is shown in Fig. 5. The rear fixed mirror (M2) is in a grove attached with cyanoacrilate adhesive. 
The front mirror (M1) is attached to the piezoelectric cylinder (PZT) with epoxy. Between the Invar steel mounting tube and the PZT ceramics cylinder, a thin plastic-shim angular ring is placed to insure that the nickel electrodes of the PZT will not be shorted by touching the steel tube. The shim is attached to the Invar by cyanoacrilate adhesive. The PZT cylinder is attached to the shim and to the Invar via epoxy. There is a section of the shim material (1/8th of the ring) that is missing and hence the epoxy over this section is directly attached to the Invar. The wires to the PZT cylinder were attached to the nickel coated walls using Sn-62 solder. The steel parts are milled Free-Cut Invar 36 Alloy which is a low thermal expansion alloy, and allow a threaded-screw displacement for setting the initial separation of the mirrors.

\subsection{Thermally-tunable FPS}

The thermally-controlled design uses a $50 \mathrm{~mm}$-long glass cylinder between the confocal interferometer mirrors (Fig. 7, upper panel). Initially an all Zerodur glass cylinder was considered with a thermal coefficient of expansion of $C_{\mathrm{TE}}=0.05 \mathrm{ppm} / \mathrm{C}$. Using a thermal system that controls the temperature to $\Delta T=$ $0.1 C$, implies controlling the spacing by steps of $\Delta e=e C_{\mathrm{TE}}$ $\Delta T=50 \mathrm{~mm} \cdot 0.05 \times 10^{-8} \times 0.1=0.0025 \mathrm{~nm}$. However, for a system working in the IR at $\lambda_{\mathrm{IR}}$ (or in the visible at $\lambda_{V}$ ) this change represents a change in the filter wavelength of $\delta \lambda=4 \delta e / \mathrm{m}$; note that $\delta e$ refers to a small displacement associated with a wavelength change $\delta \lambda$ and $\Delta e$ is a small displacement associated with a temperature change $\Delta T$. The offset order $\hat{m}$ is given by $\hat{m}=4 e / \lambda$, hence $\delta \lambda=(\delta e / e) \lambda=5 \times 10^{-9} \lambda$ and for $\lambda=\lambda_{\mathrm{IR}}$ (or $\lambda=\lambda_{V}$ ), then $\delta \lambda_{\mathrm{IR}}=7.8 \times 10^{-5} \mathrm{pm}\left(\right.$ or $\left.\delta \lambda_{V}=3.1 \times 10^{-5} \mathrm{pm}\right)$ which is quite small and implies prohibitively large changes in temperature. The $F W H M$ is given by $F S R, \lambda^{2} /(4 e)$, divided by the finesse $F$. Hence, with the refractive index $n=1$, the intensity profile $F W H M$ is $\Delta \lambda_{F W H M}=\lambda^{2} /(4 e F)$. For $R \sim 0.90$, the confocal finesse is $F \sim 15 ; \Delta \lambda_{F W H M} \sim 0.8 \mathrm{pm}$ for $\lambda_{\mathrm{IR}}$; and $\Delta \lambda_{F W H M} \sim 0.13 \mathrm{pm}$ for $\lambda_{V}$. Again, $\delta \lambda$ needs to be some fraction of the $F W H M$ in order to measure the line profile, i.e., $\delta \lambda / \Delta \lambda_{F W H M}<1$, but $\delta \lambda / \Delta \lambda_{F W H M} \sim 7.8 / 0.8=9.7$ for $\lambda_{\mathrm{IR}}$ (or $\delta \lambda / \Delta \lambda_{F W H M} \sim 3.1 / 0.13 \sim 24$ for $\lambda_{V}$ ). Hence, with Zerodur, the spectral line profile is unresolved by thermal tuning for reasonable total changes in temperature. Therefore, the thermal control design was refined.

The refinement was produced by employing a dual-glass cylinder (BK7 and Zerodur) separating the spherical mirrors, where the thermal expansion of the glass cylinder controls the inter-etalon spacing. Assuming a set of characteristics for the $F W H M$ and FSR at $\lambda_{V}$ and $\lambda_{\mathrm{IR}}$ and assuming that at least 5 points are needed across the $F W H M$ to define the profile sufficiently, then Table 2 was generated assuming that the thermal control resolution is $0.1 \mathrm{C}$. Using $(\delta e / e)=\left(C_{\mathrm{TE}} \Delta T\right)=(\delta \lambda / \lambda)$ and with the assumption that set $\delta \lambda_{V}=0.02 \mathrm{pm}$, i.e. the step size, and $\delta \lambda_{\mathrm{IR}}=0.08 \mathrm{pm}$ for a change of $\Delta T=0.1 \mathrm{C}$, then $(\delta e / e)_{V}=\left(0.02 \times 10^{-3} / 630.2\right)=3.17 \times 10^{-8},(\delta e / e)_{\mathrm{IR}}=(0.08 \times$ $\left.10^{-3} / 1564.85\right)=5.1 \times 10^{-8}$ for $\Delta T=0.1 \mathrm{C}$. Therefore, with a change of $\Delta T=1.0 \mathrm{C}$, we have the thermal coefficient of expansion requirements for the respective wavelengths: $C_{\mathrm{TEV}}=(\delta e / e)_{V}=3.17 \times 10^{-7}$ and $C_{\mathrm{TEIR}}=(\delta e / e)_{\mathrm{IR}}=5.1 \times 10^{-7}$. Using a segment of length of $L_{1}$ of Zerodur (denoted by Z) with its TCE $C_{\text {TEZ }}=5 \times 10^{-8}$ and a segment of length $L_{2}$ of BK7 with $C_{\text {TEBK7 }}=7.1 \times 10^{-6}$ then we can obtained the correct overall CTE by solving: $L_{1} C_{\mathrm{TEZ}}+L_{2} C_{\mathrm{TEBK} 7}=\left(L_{1}+\mathrm{L}_{2}\right) C_{\mathrm{TEV}}$ for $630.2 \mathrm{~nm}$. Hence if the overall length is $L=L_{1}+L_{2}$ then $L_{1}\left(C_{\mathrm{TEZ}}-C_{\mathrm{TEV}}\right)=\left(L-L_{1}\right)\left(C_{\mathrm{TEV}}-C_{\mathrm{TEBK} 7}\right)$ or $L_{1}=L$ $\left(C_{\mathrm{TEV}}-C_{\mathrm{TEBK} 7}\right) /\left(C_{T E V}-C_{\mathrm{TEBK} 7}\right)$. For $L_{1} / L=\left(3.17 \times 10^{-7}-\right.$ $\left.7.1 \times 10^{-6}\right) /\left(5.0 \times 10^{-8}+7.1 \times 10^{-6}\right)=0.962$ and for $L=50 \mathrm{~mm}$,
Table 2. Initial thermal design requirements with $R=0.9$.

\begin{tabular}{lll}
\hline \hline & $\lambda_{V}=630.2 \mathrm{~nm}$ & $\lambda_{\mathrm{IR}}=1564.85 \mathrm{~nm}$ \\
\hline$F W H M$ & $0.10 \mathrm{pm}$ & $0.40 \mathrm{pm}$ \\
$F S R$ & $2 \mathrm{pm}$ & $15 \mathrm{pm}$ \\
$\begin{array}{l}\text { Step Size } \\
\text { (at 5pts/FWHM) }\end{array}$ & $0.02 \mathrm{pm}$ & $0.08 \mathrm{pm}$ \\
$\begin{array}{l}\text { Number of points to } \\
\text { cover the full } F S R\end{array}$ & 100 & 188 \\
$\begin{array}{l}\text { Change in temperature } \\
\text { required to cover } F S R\end{array}$ & $10 \mathrm{C}$ & $19 \mathrm{C}$ \\
\hline
\end{tabular}

then $L_{1}=48.1 \mathrm{~mm}$ for Zerodur and $L_{2}=1.89 \mathrm{~mm}$ for BK7. Therefore by introducing a $1.89 \mathrm{~mm}$ segment of BK7, the combination provides the correct over all CTE need for the visible. The values to meet the correct CTE for the IR needs to be $L_{1}=46.74 \mathrm{~mm}$ and $L_{2}=3.26 \mathrm{~mm}$ which was the final design specifications, since they also satisfy the visible requirement. The electric requirement for $20 \mathrm{C}$ temperature range and controlling the individual tripod of legs to $0.1 \mathrm{C}$ is provided by a temperature transducer (type AD590) with a heater strip. The measured time constant for the constructed interferometer was $360 \mathrm{~s}$. The resulting temporal sensitivity is too long for solar observations; however the dual-glass cylinder has no associated hysteresis.

\section{Alignment}

The parallel plates of a FPP must be exactly parallel, i.e., to within $\lambda / \mathrm{D} \sim 10^{-6}$ radians. Conversely, because of the spherical shape of the FPS, the alignment of the centers of the mirrors needs to be only roughly aligned, to within about $0.1 D / r \sim 10^{-2}$ radians. This allows the axial alignment to be glued in place with no further improvement in tilt. However, for the axial separation of the confocal mirrors, the precision is more critical than the axial alignment and is given by $|\epsilon|=\left(\lambda r / 32 F_{\mathrm{R}}\right)^{1 / 2}$, hence, for $r=50 \mathrm{~mm}, \lambda=1.564 \mu \mathrm{m}, F=15,|\epsilon|=13 \mu \mathrm{m} \sim 8 \lambda$ (Vaughan 1989, p. 195). However, setting the initial separation is straight forward (Hercher 1968), and is based on the fringe movement about the confocal separation. The fringe pattern radius for fringe orders $\hat{m}=[-10,10]$ is plotted versus the departure from the confocal position in Fig. 6 . The thick solid line is for $\hat{m}=0$. The dotted line is the maximum radial dispersion curve $(d \rho / d \lambda)$. For scale, a rectangle is defined by the spot radius of $\rho_{\mathrm{s}}$ and departure of $w= \pm 100 \lambda$. The wavelength is for the photospheric visible Zeeman sensitive line $\left(\lambda_{V}=630.2 \mathrm{~nm}\right)$. Spot radius $\rho_{\mathrm{s}}$ is defined when the actual $F W H M$ is $\Delta \lambda_{\text {actual }}=1.42 \Delta \lambda_{F W H M}$. If the confocal departure is less than the confocal distance $(e<0)$, as $|e|$ is decreased, the low order fringe pattern moves outward and for $e>0$, as $|e|$ is increased the fringes move inwards. For the piezoelectric tuned FPS, a fine screw adjustment allowed the confocal separation adjustment. For the thermally tuned FPS, the glass tube was carefully polished and checked for the proper separation and then optically contacted to the mirrors.

\section{Test results}

The confocal interferometer sub-picometer test results are given in terms of profile line scanning and imaging. In Fig. 8, the results are shown using the piezoelectric-controlled FPS. The scans show the hysteresis effect of the lead zirconate titanate cylinder (cf. Crawford 1961). A Powell multiple parameter least-squares fit of the scans determined that the sub-picometer 


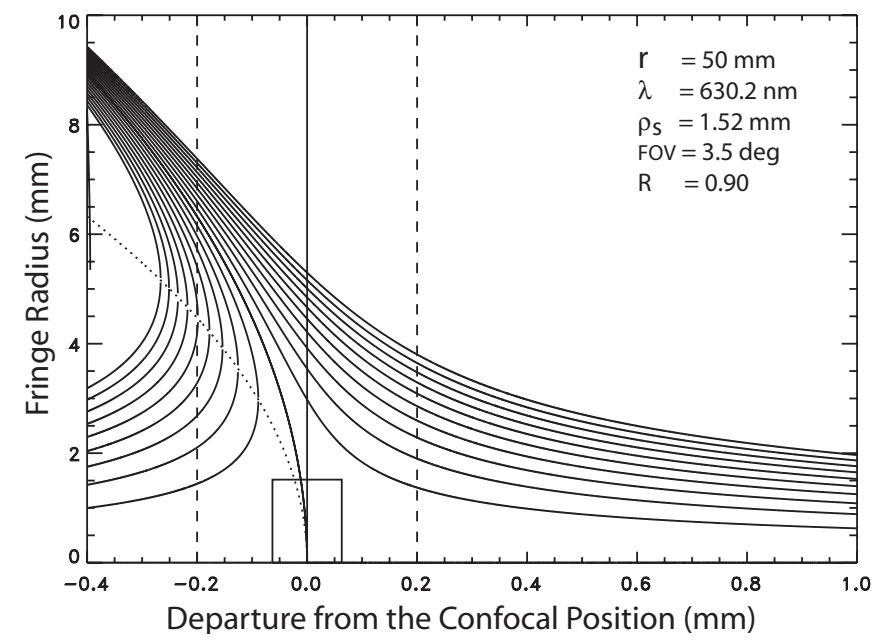

Fig. 6. Near confocal Fabry-Pérot fringe patterns. The curves give the radii of the circular interference pattern for collimated light in the paraxial approximation.

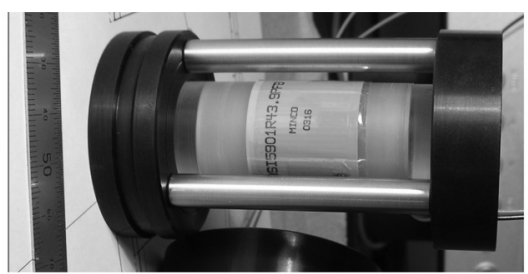

Thermally Controlled

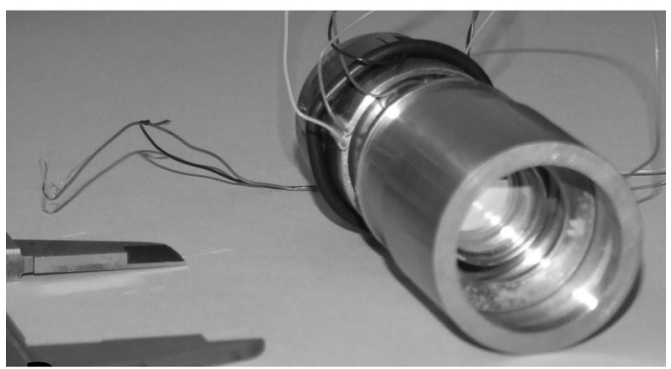

Piezoelectric Controlled

Fig. 7. The two constructed confocal interferometers with subpicometer spectral resolution. For each, the confocal distance is $50 \mathrm{~mm}$ and the aperture is $25 \mathrm{~mm}$.

spectral resolution was $F W H M=0.33$ pm with $F S R=1.99 \mathrm{pm}$, $F=6.0$, and effective reflectivity of 0.77 . These acceptable values include the effect of complete optical configuration and hence the finesse is lower than the theoretical value.

Figure 9 shows the imaging capability of the confocal interferometer. An US Air Force Resolution Chart is imaged at $\lambda=630.2 \mathrm{~nm}$. For this large field of view, the two images of the FPS are captured by the CCD camera, and the effects of spherical aberrations can be seen at the outer edge of the image. The center of the optical axis is near the center of the figure. For this magnification, the resolution is limited by the CCD.

Employing larger magnification with a zoom lens, Fig. 10 shows the center of USAF resolution charts imaged employing an entrance mask with an aperture of $2 \rho_{\mathrm{s}}=2 r(\lambda / r F)^{1 / 4}=$ $3.8 \mathrm{~mm}$ were $F=6$ and $\lambda=630.2 \mathrm{~nm}$. The image was formed using an negative pattern, 1951 USAF resolution chart (Edumund Optics NT38-256, maximum resolution $228 \mathrm{lp} / \mathrm{mm}$, i.e., Group 7/Element 6) using a $175 \mathrm{~mm}$ focal length lens with

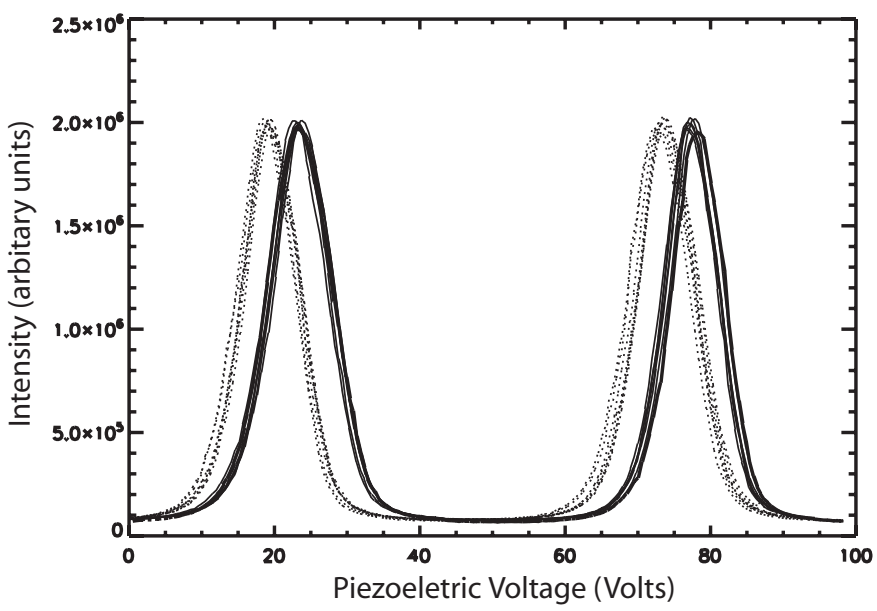

Fig. 8. Piezoelectric-controlled FPS scans of the HeNe laser line. The data is partially corrected for thermal drift. The solid lines are scans with the PZT voltage increasing and the dotted lines are with voltage decreasing. The separation of profiles set shows the hysteresis effect which can be corrected.

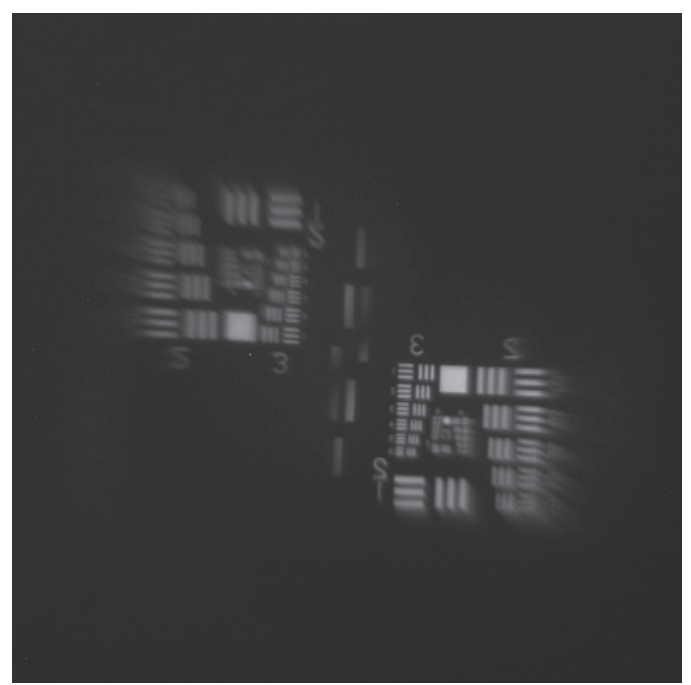

Fig. 9. An image of part of an Air Force Resolution Chart imaged at $\lambda=630.2 \mathrm{~nm}$ through the FPS. Both exit images $\left(S_{1}^{\prime}\right.$ and $\left.S_{2}^{\prime}\right)$ of the FPS are seen.

the image and object distances equal $(350 \mathrm{~mm})$. The $4 \mathrm{~mm}$ aperture was slightly offset from the optical axis. The FOV is $2.5 \mathrm{deg}$. With this optical configuration, the spatial resolution is only slightly degraded, $\sim 11 \%$, as seen by comparing the two images and by comparing the images with the diffraction limit of a $4 \mathrm{~mm}$ aperture, which is at the frequency giving by Group 4/Element 4 of the resolution chart. These test result show that a FPS allows imaging interferometry at high spectral resolution.

\section{Conclusions}

The confocal interferometer sub-picometer $F W H M$ spectral filters for visible and near-IR solar magnetography and Dopplergraphy will lead to a better understanding of the magnetic field and advective flow gradients through the solar atmosphere and links observation between different spectral lines at various heights. For high spectral resolution, a confocal (also called a spherical or Connes) interferometer enhances the scientific value of future solar satellite missions by increasing the field of view and throughput of magnetographs, decreases the 


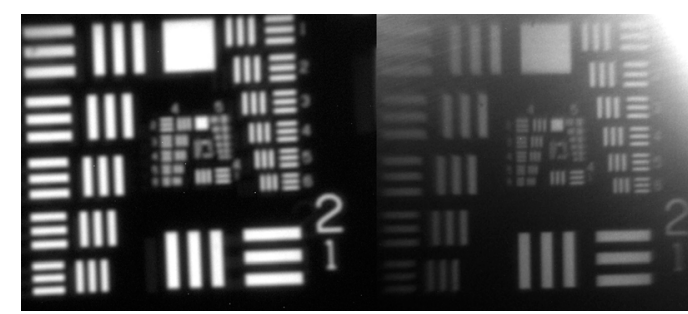

Fig. 10. Images of an USAF resolution Chart at $\lambda_{\mathrm{V}}$ without (left) and with (right) the FPS. The FPS was masked with an aperture of $2 \rho_{\mathrm{s}}$. The degradation to the MTF function is less than $11 \%$. A white light source and narrow passband filter $\left(\left(\Delta \lambda_{F W H M}=30 \mathrm{~nm}\right)\right.$ was employed. Multiple orders were passed to have minimum laboratory exposure times. The image through the FPS (right) has straylight as a result of the longer exposure time.

cost of the mission by allowing smaller optical components, and increases the mission probability of success by relaxing optical tolerances of the interferometer. We have developed, tested, and demonstrated the imaging capabilities of a very narrowpassband confocal interferometer. The FOV and spatial resolution of a FPS is set by the diffraction limit of the confocal spot size. The spectral resolution is set by the adjustment of the confocal separation. The FPS can be use for imaging and provides sub-picometer spectral resolution. The high throughput of the FPS as compared to the FPP makes this interferometer the choice for obtaining high resolution solar line profiles.

For future studies, there are two imaging FPP instruments that can be used as prefilters for our FPS research on solar phenomena. In the visual and the near IR, the NSO Sacramento Peak's high-resolution dual Queensgate Fabry-Pérot interferometers can be employed, with the narrowest passband compatible with the FPS's FSR. At $\lambda_{V}=630.2 \mathrm{~nm}$ the NSO's $50 \mathrm{~mm}$ etalons ET50-1001 $(F W H M=9.0 \mathrm{pm}, F S R=327 \mathrm{pm})$ and ET50-1046 $(F W H M=1.7 \mathrm{pm}, F S R=72.1 \mathrm{pm})$ can be employed. In the near IR, the etalons ET50-993 $(F W H M=11.3 \mathrm{pm}, F S R=522.5 \mathrm{pm})$ and ET50-998 $(F W H M=168.3 \mathrm{pm}, F S R=3932 \mathrm{pm})$ could be employed as a prefilter for the FPS. For a S/N of 500, a 15point $\lambda_{\mathrm{IR}}$ spectral profile scan time is estimated to be $40 \mathrm{~min}-$ utes using the Dunn Solar Telescope. Also for the NIR, the New Jersey Institute of Technology/Big Bear Solar Observatory's Near Infrared Magnetograph (NIRM) could be employed which has a FPP with $F W H M=8.8 \mathrm{pm}$ and $F S R=548.7 \mathrm{pm}$. We plan to employ these systems to obtain sub-picometer spectral resolution Stokes $I$ and $V$ line profiles in the umbral regions to determine the vertical magnetic field and velocity gradients across the height of formation of the spectral lines FeI $\lambda_{\mathrm{V}}$ and $\lambda_{\mathrm{IR}}$. However, the final spectral information will degraded by the relatively long integration times.

With the construction of ground-based solar telescopes with aperture larger than a meter and the planned National Solar Observatory's 4-m Advanced Technology Solar Telescope, the observed transverse spatial resolution will be less than the half-width of the vertical height of formation of the observed spectral lines. Using a confocal sub-picometer interferometer, the observations can be made of the same spectral line to produce vertical height information compatible to the transverse scale within a time frame consistent with the resolution. These observations can be supplemented with multiple line observations; however, multiple line analysis relies on the assumption that the height of formation of the atomic levels can be directly related to the height of formation of the observed line. The radiometry calculations given in the introduction confirm that such observations can be made, and we argue that these should be pursued. With the completion of the future observations listed in the last paragraph, the usefulness of the observations will be tested.

Acknowledgements. We would like to thank D. Budker (UC Berkeley), E. Bickel (Channel Industries), and N. Benoit (Boston Piezo Optics) for their assistance on the design and mechanical construction of the PZT interferometer. We thank J. Sanchez Almeida (IAC), L. Bellot Rubio (IAA), J. Bruls (KIS), and W. Cao (NJIT) for there comments and calculations on the possibilities of high spectral resolution in the near infrared. We would like thank the V. Martinez Pillet for helping to greatly clarify the manuscript through a careful reading of the paper. This research was supported by the Marshall Space Flight Center's Institutional Research and Development Program.

\section{References}

Allen, C. W. 1973, Astrophysical Quantities (London: Athlone Press), 165 Balasubramaniam, K. S., \& Uitenbroek, H. 2001, NSO ATST Technical Note 2, High Spectral Resolution Requirements, Tucson, AZ

Bernasconi, P. N., Rust, D. M., Murphy, G., \& Eaton, H. A. C. 1999, ASP Conf. Ser., 183, 279

Bettonvil, F. C. M., Hammerschlag, R. H., Sütterin, P., et al. 2004, SPIE Conf., $5489,34,362$

Budker, D., Orlando, D. J., \& Yashchuk, V. 1999, Am. J. Phys., 67, 584

Bruls, J. H. M. J., \& Lühe, O. V. D. 2001, A\&A, 366, 281

Clark, J. A. 1972, Opt. Comm., 5, 163

Connes, P. 1958, J. Phys. Rad., 19, 262

Crawford, A. E. 1961, British J. Appl. Phys., 12, 529

Damé, L. 1994, Proc. SPIE, 2200, 35

Davis, J. M., West, E. A., Moore, R. L., et al. 2005, Proc. SPIE, 5901, 273

Fabry, C., \& Pérot, A. 1902, ApJ, 15, 73

Gary, G. A., Balasubramaniam, K. S., \& Sigwarth, M. 2003, Proc. SPIE, 4853, 252

Hercher, M. 1968, Appl. Opt., 7, 951

Hammerschlag, R. H., von der Lühe, O., Bettonvil, F. C. M., Jägers, A. P. L., \& Sink, F. 2004, Proc. SPIE, 5489, 491

Johnson, J. R. 1968, Appl. Opt., 7, 1061

Keil, S., Oschmann, J. M. Jr., Rimmele, T. R., et al. 2004, Proc. SPIE, 5489, 625

Maltby, P., Avrett, E. H., Carlsson, M., et al. 1986, ApJ, 306, 284

Mathew, S. K., Lagg, A., Solanki, S. K., et al. 2003, A\&A, 410, 695

Lites, B. W. 2001, The SUNRISE Balloon Investigation of Solar Magnetic Fine, Structure, Dynamics, and Energy Transport, Accept Proposal, NASA NRA

Rouppe van der Voort, L. H. M., Löfdahl, M. G., Kiselman, D., \& Scharmer, G. B. 2004, A\&A, 414, 717

Rouppe van der Voort, L. H. M., Hansteen, V. H., Carlsson, M., et al. 2005, A\&A, 437, 327

Sanchez Almeida, J. 2006, private communication

Schlichenmaier, R. 2006, Prospects of Solar Physics from the Ground, Solar Activity and its Magnetic Origin, Proc. IAU Symp., 233, ed. A. A. Hady, \& V. Bothmer

Solanki, S. K., Rüedi, I., \& Livingston, W., Å. 1992, A\&A, 263, 312

Stein, R. F., \& Nordlund, A. 2006, ApJ, 642, 1246

Stenflo, J. O., Harvey, J. W., Brandt, J. W., \& Solanki, S. 1984, A\&A, 131, 333

Vaughn, J. M. 1989, The Fabry-Pérot Interferometer: History, Theory, Practice, and Applications (Philadelphia: Adam Hilger) 